\title{
La licenciatura en Turismo Alternativo de la Universidad Intercultural Maya de Quintana Roo (UIMQROo), desde la mirada intercultural de los egresados
}

\section{The Degree in Alternative Tourism from the Universidad Intercultural Maya de Quintana Roo (UIMQROO), from the Intercultural Perspective of Graduates}

\author{
Cecilia del Socorro Medina Martín \\ Universidad Intercultural Maya de Quintana Roo (uimQroo), México \\ cecilia.medina@uimqroo.edu.mx \\ Margarita de Abril Navarro Favela \\ Universidad Intercultural Maya de Quintana Roo (uimQroo ), MÉxico \\ margarita.navarro@uimqroo.edu.mx \\ Andrea Danilu Uc Angulo \\ Universidad Tecnológica Metropolitana (UTm ), México \\ andrea.uc.angulo@gmail.com
}

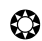

RESUMEN

Para evaluar la pertinencia de la licenciatura en Turismo Alternativo de la Universidad Intercultural Maya de Quintana Roo (UIMQROo), en marzo de 2018 las profesoras de este programa educativo trabajamos con egresados mediante la metodología sociocrítica ${ }^{1}$ y analizamos sus percepciones, expresadas tanto en un cuestionario individual como en un grupo focal. En ambos casos, se plantean cambios que responden a las competencias requeridas en las demandas laborales del turismo alternativo, a la vez que reconocen, por tener una mirada intercultural, la importancia de seguir incorporando los saberes tradicionales y de fortalecer la identidad con base en la lengua y la cultura maya. Asimismo, los egresados seńalan la relevan-

\footnotetext{
${ }^{1}$ El método sociocrítico se caracteriza por hacer partícipes a los involucrados en el empoderamiento social, es decir, el investigador sólo es un facilitador e implica la adopción de decisiones consensuadas para la transformación desde el interior. Todas estas características son tomadas del paradigma estudiado por Philipp (1998). También implica el empleo de herramientas participativas para transformar la realidad social y el intercambio de conocimiento como apropiación social.
}

40 - La licenciatura en Turismo Alternativo de la Universidad Intercultural Maya de Quintana Roo (UIMQROO)... 
cia del respeto a la diversidad, lo cual revaloriza la pertinencia y el aporte social del modelo educativo intercultural con énfasis en la vinculación comunitaria.

Palabras clave: Interculturalidad; modelo educativo intercultural; licenciatura en Turismo Alternativo; UIMQRoo; egresados.

\begin{abstract}
To evaluate the relevance of the degree in Alternative Tourism Universidad Intercultural Maya de Quintana Roo (UIMQROO), in March 2018, teachers of the educational program worked with the graduates using the social-critical methodology. Teachers analyze the perceptions of the graduates, expressed in an individual questionnaire and a focus group. In both cases, they proposed changes that respond to the skills required in the labor demands of alternative tourism. In turn, graduates acknowledged, having an intercultural outlook, the importance of continuing to incorporate traditional knowledge and strengthening identity based on the Mayan language and culture. They also note the importance of respect for diversity, which enhances the relevance and social contribution of the Intercultural Educational Model with an emphasis on community involvement.

Keywords: Interculturality; Intercultural Educational Model; Degree in Alternative Tourism; UIMQROO; Graduates.
\end{abstract}

Fecha de recepción: 01/11/2019

Fecha de aceptación: 28/01/2020

\section{Contexto de la Universidad Intercultural Maya de Quintana Roo (UIMQROO)}

El modelo educativo intercultural y bilingüe inició en México en el año 2001 con la creación de la Coordinación de Educación Intercultural y Bilingüe (CGEIB) de la Secretaría de Educación Pública (SEP). Actualmente son 11 Universidades Interculturales (UI) distribuidas en todo el territorio nacional, cuyo modelo educativo a nivel superior pretende atender las necesidades de las poblaciones indígenas, aprovechando las diferencias entre culturas para enriquecer las posibilidades de desarrollo del país (Casillas \& Santini, 2009), así como evitar los problemas de la educación segregada, al abrirse a personas indígenas y no indígenas. De esta forma, se ofrece una educación encaminada a fortalecer las lenguas y culturas que definen a México como un país pluricultural (Schmelkes, 2008).

La interculturalidad no significa coexistir, sino convivir entre grupos culturales distintos con vínculos de respeto y desde planos de igualdad, con la intención de explicitar y cuestionar las formas en que se relacionan las culturas. Por esta razón, toda la población debe estar involucrada (Saldívar, 2006).
En esta aspiración de vivir sin diferencias, la interculturalidad es parte de un proyecto de nación en el que no cabe el racismo y se promueve la tolerancia, el respeto y el aprecio. Por esto, tomando como premisa este planteamiento, coincidimos con que "debemos perseguir en nuestra actividad educativa con toda la población, incluyendo en especial la mestiza" (Schmelkes, 2013, p. 7).

Con base en los principios mencionados, la educación intercultural pretende enriquecer de manera personal a los estudiantes fomentando la interacción y la convivencia con otras culturas (Sánchez, 2016), valorando las diferencias. Como señala Fernández (1995), la escuela es una extensión de la familia, ya que ambas son depositarias de valores que determinan la trayectoria de los individuos en la sociedad. Sin embargo, también puede ser una plataforma para la lucha revolucionaria, en la que hay poder y conflicto de intereses, los cuales son las claves del paradigma sociocrítico.

En el año 2006, se creó la Universidad Intercultural Maya de Quintana Roo (UIMQROo), ubicada en la carretera Muna-Felipe Carrillo Puerto kilómetro 137 en la localidad de La Presumida, en el mu- 
nicipio de José María Morelos, Quintana Roo. En esta universidad se imparten ocho programas de licenciatura y una maestría. En su misión se describe:

[...] contribuir al desarrollo socioeconómico del estado de Quintana Roo y la región, mediante la formación de profesionistas [...] y la generación y aplicación de conocimiento innovador; a través de un modelo educativo intercultural, multilingüe y con una fuerte vinculación entre los diferentes sectores (UIMQROO, 2016).

La definición de modelo educativo adoptado en la UIMQROO es la operacionalidad de un arreglo de elementos que representa las teorías, los conceptos, los principios, los valores, la filosofía y la visión, que sustentan su misión. La operacionalidad forma parte de un sistema, de un arreglo institucional, que hace viable el modelo que permite su evaluación y mejora continua (UIMQROo, 2010).

Como parte del modelo intercultural se contemplan elementos importantes para su funcionamiento, como el Sistema Iknal, más que tutoría académica (Navarro, 2015); planes de estudio flexibles, programas educativos integrados (licenciatura y posgrado) y modelo de titulación progresiva para lograr la formación integral de los estudiantes (Ver Figura 1) (UIMQROO, 2010).

Figura 1

Representación esquemática del modelo educativo (UIMQROO, 2010, p. 26)

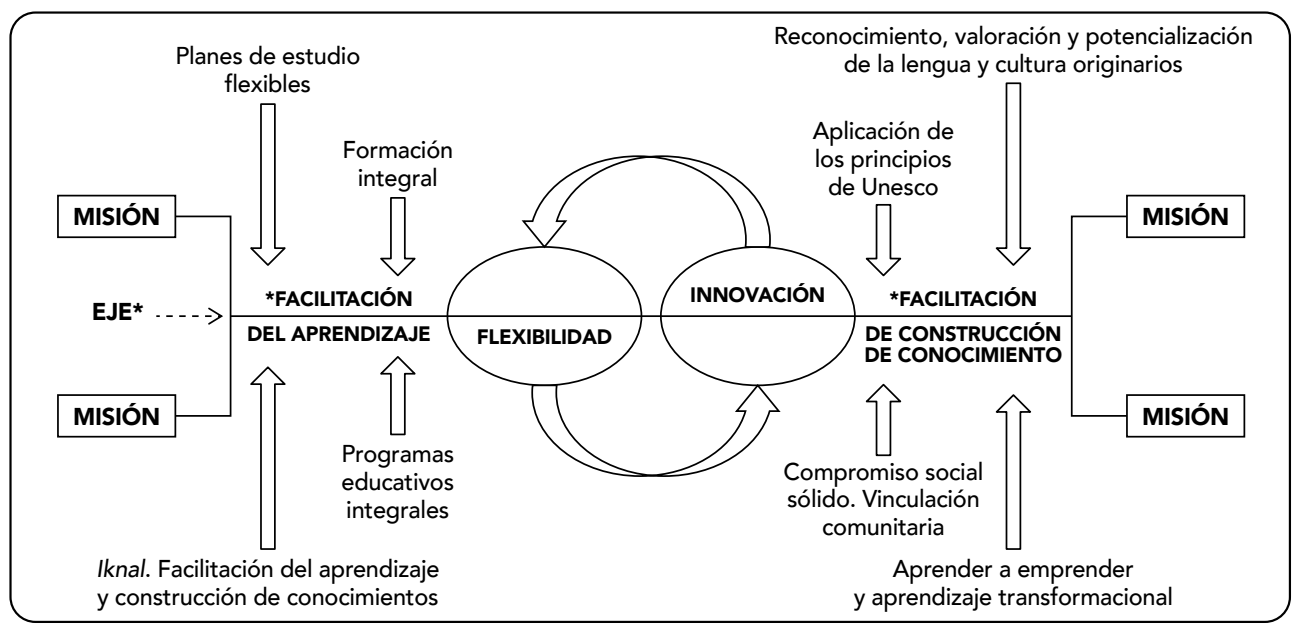

El Programa Educativo (PE) de la licenciatura en Turismo Alternativo se oferta desde la fundación de la UIMQROo. En el año 2011 obtuvo la acreditación de los Comités Interinstitucionales para la evaluación de la Educación Superior (CIEEs), en el nivel I, y se evaluó de nuevo en 2019, por lo que desde 2018 los profesores se dieron a la tarea de crear un instrumento con indicadores que permitieran alcanzar el objetivo de la autoevaluación.

Debido a la continua diversificación del turismo es necesario que el PE de esta licenciatura forme profesionales con las competencias necesarias para afrontar los retos de la demanda laboral y para incidir en su contexto económico, político y cultural, con base en su lengua y cultura local. Por tales motivos, el PE parte de la necesidad de formar a un profesionista que aproveche de manera sostenible y con responsabilidad social el patrimonio natural y cultural, como producto de la sinergia entre sus conocimientos ancestrales, como las prácticas productivas tradicionales, las manifestaciones culturales y los conocimientos propios de la disciplina.

\section{Modelo de competencias: enseñanza-aprendizaje}

Las competencias son una combinación de conocimiento, capacidades y características personales, valores, habilidades, entre otras, que permiten demostrar lo aprendido en el proceso de enseńanza a

42 - La licenciatura en Turismo Alternativo de la Universidad Intercultural Maya de Quintana Roo (UIMQROO)... 
través de la capacidad para realizar algo. Toda competencia debe mostrar un resultado, por lo que se clasifican en básicas, genéricas y específicas; éstas últimas son propias en una ocupación o profesión (García Retana, 2011).

Las competencias están estrechamente vinculadas con el modelo educativo intercultural, ya que bajo este enfoque se pretende que los profesores y los estudiantes contribuyan al desarrollo socioeconómico de la región a través de la implementación de proyectos identificados en los diagnósticos de los Trabajos de Vinculación Comunitaria (TVC). Los estudiantes aprenden y los profesores enseñan bajo el modelo de competencias saber-expresar-hacer. De este modo, se fortalece el desarrollo profesional y posteriormente esto genera que los estudiantes tengan experiencias que les permitan incorporarse al mundo laboral (UIMQROo, 2015).

\section{La evaluación del Programa Educativo de Turismo Alternativo (PETA), considerando el enfoque intercultural y la vinculación comunitaria}

Evaluar la aplicación práctica del modelo intercultural en la adquisición de competencias y en la inserción laboral no es tarea fácil; sin embargo, a 13 años de implementarse, se cuenta con datos suficientes.

Partiendo de lo anterior, en el año 2018 nos reunimos 23 egresados y tres profesoras para analizar el PE de la licenciatura en Turismo Alternativo. Se trabajó con el método sociocrítico enfocado en la transformación de las estructuras de la realidad social, para dar respuesta a situaciones con base en la autorreflexión de los participantes (Maldonado, 2018). En el ámbito de la educación, este método pretende tener una visión global e interpretativa, que procure la transformación del estilo de aprendizaje, a través de la generación de una conciencia de la realidad, basada en experiencias prácticas y reflexiones que den paso a un criterio propio (Alvarado \& García, 2008). El propósito de las ui es extender las oportunidades educativas para atender a estudiantes de diversos orígenes, lenguas y culturas del país (Casillas \& Santini, 2009).
Utilizamos dos herramientas: una cuantitativa, que consistió en un cuestionario administrado individualmente para evitar que las respuestas se influenciaran con las opiniones de los otros participantes; $y$ una cualitativa mediante un grupo focal con la técnica "lluvia de ideas".

Debido a que se trató de un proceso de autorreflexión y análisis de los egresados a partir del conocimiento adquirido en su formación bajo el modelo intercultural y las experiencias adquiridas en el ámbito laboral (teoría y práctica), nos propusimos trabajar con nueve categorías del modelo intercultural de la universidad (UIMQROo, 2016).

Las categorías de análisis fueron:

1. Datos de identificación y perfil de egreso

2. Objetivo general y competencias profesionales de la licenciatura; la importancia del Taller de Vinculación Comunitaria

3. Contenidos de aprendizaje (contestar el cuestionario, presentación del currículo y trabajo en equipo con las propuestas por asignatura por semestre)

4. Proceso de enseñanza-aprendizaje

5. Procedimiento de evaluación

6. Perfil del docente (trabajos en equipo para discutir y proponer situaciones ideales de los temas abordados)

7. Taller de vinculación con la comunidad

8. Servicio social

9. Intercambio académico

\section{Datos de identificación y perfil de egreso}

El perfil de egreso es el conjunto de competencias y todos los atributos que el estudiante posee cuando concluye su educación escolar que le permitirán desempeñarse con éxito en el ámbito laboral. Evaluar este perfil permite ver la pertinencia del PE y debe corresponder a las necesidades tanto de los empleadores como del saber ser.

Para evaluar si estas competencias y valores son aplicables en el campo laboral de los egresados, se diseñó un cuestionario que aportó datos generales de cada participante para obtener características de 
la muestra, de la generación en que estudió y otros datos como: edad, ocupación actual y grado de estudio. También se incluyó una subcategoría de información laboral como indicador socioeconómico. Los resultados del cuestionario fueron analizados en una base de datos Excel $^{\circledR}$ versión 2017. Por otra parte, la información de las preguntas abiertas fue sistematizada a través de categorías de análisis para agrupar las respuestas de los egresados, también se delimitaron subcategorías, considerando los porcentajes de las respuestas que se repitieron.

La muestra se conformó de los egresados que acudieron a la invitación de la UIMQROO, con un total de 14 hombres y nueve mujeres $(\mathrm{N}=23)$. En cuanto a la representatividad por generación, de la sexta y séptima sólo acudieron un graduado de cada una, mientras que de la quinta no acudió nadie, de la cuarta fueron dos, de la tercera tres, de la segunda cuatro y de la primera 12 , siendo esta generación la de mayor participación en el estudio.

La mayoría de los egresados participantes (70\%) tiene el grado de licenciatura, mientras que sólo uno se tituló como profesional asociado (4\%); dos tienen maestría concluida ( $9 \%)$, y otros cuatro (17\%) están en proceso de iniciar su posgrado.

En la categoría "campo de trabajo" se realizaron las preguntas correspondientes a su empleo actual y sus experiencias laborales previas; se obtuvo que la totalidad de la muestra se encuentra empleada. La mayoría trabaja en el área de la docencia (52\%), mientras que $26 \%$ labora en el turismo como empleados en parques temáticos y en servicios turísticos (cafeterías). A su vez, $9 \%$ son servidores públicos o dependientes en el ayuntamiento local y $13 \%$ se emplea en otras áreas, como: el Comité Estatal de Sanidad Vegetal de Quintana Roo, de sobrecargo en una naviera, en la Comisión Nacional de Áreas Naturales Protegidas como analista de áreas protegidas y en tiendas departamentales en el área de ventas y atención al cliente.

Se preguntó a los egresados cómo consiguieron el empleo: la mayoría contestó que a través de concurso por convocatoria (39\%), otros por medio de una bolsa de trabajo $(30 \%)$ y en menor cantidad por reco- mendación (13\%). Los individuos restantes no contestaron (17\%). En cuanto a la opinión o perspectiva de los empleadores, la mayoría son considerados buenos en el desempeño de sus funciones (61\%), mientras que otra parte mencionó que los consideran excelentes (35\%) y una persona no contestó (4\%).

Entre las actividades que desarrollan en su trabajo profesional, se mencionó una gran diversidad: docencia, atención al cliente, elaboración y gestión de proyectos, actividades administrativas, trabajo social, elaboración de alimentos y bebidas, guías, observación de aves, actividades de turismo de aventura, envío de muestras para análisis, capacitación de nuevo personal, operación de sistemas informáticos, programa de prevención de enfermedades, intérpretes del patrimonio, generación de ventas y seguimiento a softline y bigticket en touroperadora, así como control de inventario. Los egresados comentaron que han tenido más de un trabajo y, por lo tanto, se han desempeñado en diversas áreas.

\section{Objetivo general y competencias profesionales de la licenciatura; la importancia del Taller de Vinculación Comunitaria}

Se hicieron preguntas abiertas con la finalidad de identificar la aplicación de las competencias profesionales en las actividades de los egresados, por ejemplo, si su trabajo de titulación se relaciona con las tareas que realiza en su empleo y en qué medida. Los egresados argumentaron que en las asignaturas adquirieron diversidad de competencias y expresaron lo siguiente: estrategias para impartir clases, planeación, manejo y control de grupos; educación ambiental (monitoreo y observación de aves, formación de grupos comunitarios para el monitoreo biológico); actualización de información; consulta de diversas fuentes bibliográficas; elaboración y gestión de proyectos e interpretación cultural (arqueológica e histórica).

Por su parte, la herramienta cualitativa consistió en la investigación participativa (IAP), que coadyuva al paradigma sociocrítico (el cual pretende el involucramiento de los actores que conlleve al cambio social) al ser una herramienta en la cual los participantes

44 - La licenciatura en Turismo Alternativo de la Universidad Intercultural Maya de Quintana Roo (UIMQROO)... Cecilia del Socorro Medina Martín, Margarita de Abril Navarro Favela, Andrea Danilu Uc Angulo. DIDAC 76 (2020): 40-50 
son quienes plantean las soluciones a las problemáticas de su contexto (Melero, 2011). Mediante grupos focales y la lluvia de ideas, se expusieron las experiencias que tuvieron como estudiantes y ahora egresados. La participación fue fundamental dentro del proceso sociocrítico, pues tiene como objetivo promover las transformaciones sociales con el principio de la integración de todos los participantes (Alvarado \& García, 2008). En el estudio fue importante conocer las competencias adquiridas cuando fueron estudiantes y cómo éstas les favorecen en sus labores profesionales, dando pie así a la autorreflexión para mejorar en alguna situación de vida.

Los egresados mencionaron en la lluvia de ideas que los TVC realizados les permitieron aprender a elaborar proyectos sociales, así como a trabajar y solventar las necesidades de las comunidades. Según Maldonado (2018), la investigación sociocrítica se fundamenta en la transformación de las comunidades, lo cual contribuye al mejoramiento de la calidad de vida de los habitantes. Asimismo, la formación que adquieren los estudiantes en el modelo intercultural también contribuye a construir de manera participativa la realidad comunitaria.

En la UIMQROO se cursan los idiomas inglés, maya y español, lo cual permitió a los egresados desarrollar seguridad para compartir conocimientos en otras lenguas. La interculturalidad les provee aportaciones de los conocimientos locales en: las asignaturas que imparten, la actitud laboral, la lengua y la apreciación por la cultura.

La forma de titulación más común es la tesis (65\%), le sigue el proyecto de desarrollo comunitario $(22 \%)$ y otras dos por las que un solo egresado optó en cada una: informe de vinculación con la comunidad y un trabajo de difusión. Estos trabajos fueron derivados de los TVC (97\%), a excepción de tres casos (13\%). La mayoría de los individuos de la muestra concluyeron en tiempo y forma el proceso de titulación ( $88 \%$ ) y sólo $22 \%$ requirió mayor tiempo para titularse por causas relacionadas con la economía familiar.

Las tesis contribuyeron con las comunidades, se crearon proyectos, se motivó a continuar el trabajo

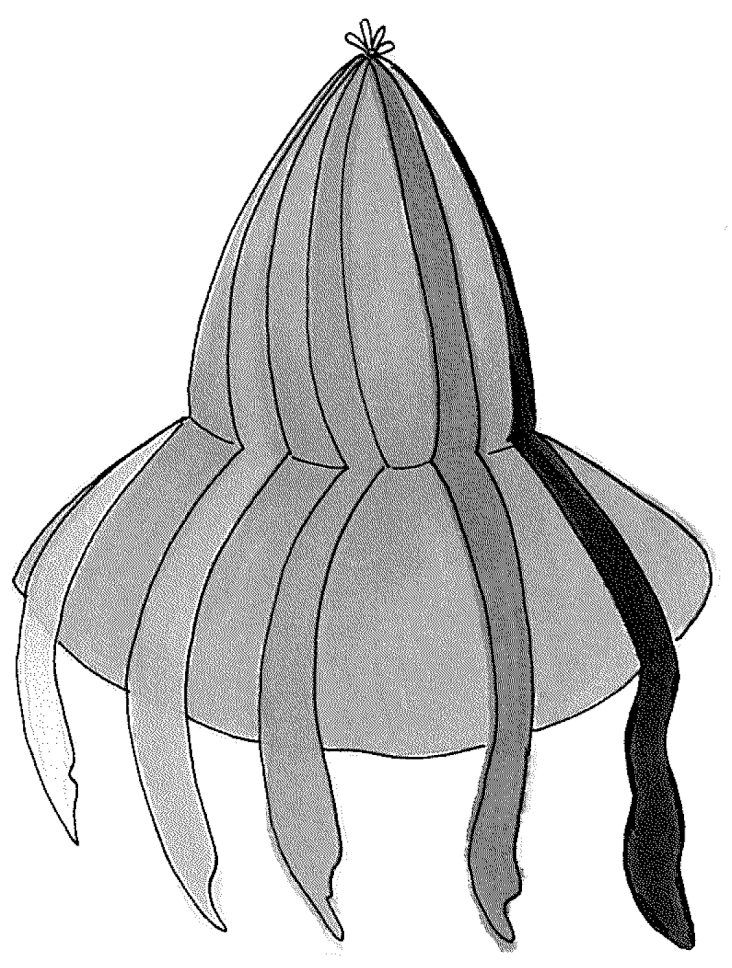

de los ya existentes — como promoción turística (vídeos, creación de logotipos y comercialización y promocionales) - y se propició la diversificación del producto. También se capacitaron en atención al cliente, atención y prevención de accidentes, preparación y capacitación en primeros auxilios e incluso para la aplicación de una certificación.

\section{Contenidos de aprendizaje}

Para evaluar la pertinencia de los conocimientos, las experiencias prácticas que se imparten y su aportación para elaborar el trabajo de titulación, se respondió que en gran medida las asignaturas, las prácticas y las salidas de campo, así como el constante asesoramiento, permitieron aplicar lo aprendido en el aula en la realización del trabajo de titulación, incluidos los cursos de formación básica, aunque reconocieron que faltó ahondar en temas de gestión de proyectos.

La opinión de los egresados acerca de las asesorías del comité para terminar su trabajo de titulación fue positiva en casi todos los casos y mencionaron que las sugerencias mejoraron el tra- 


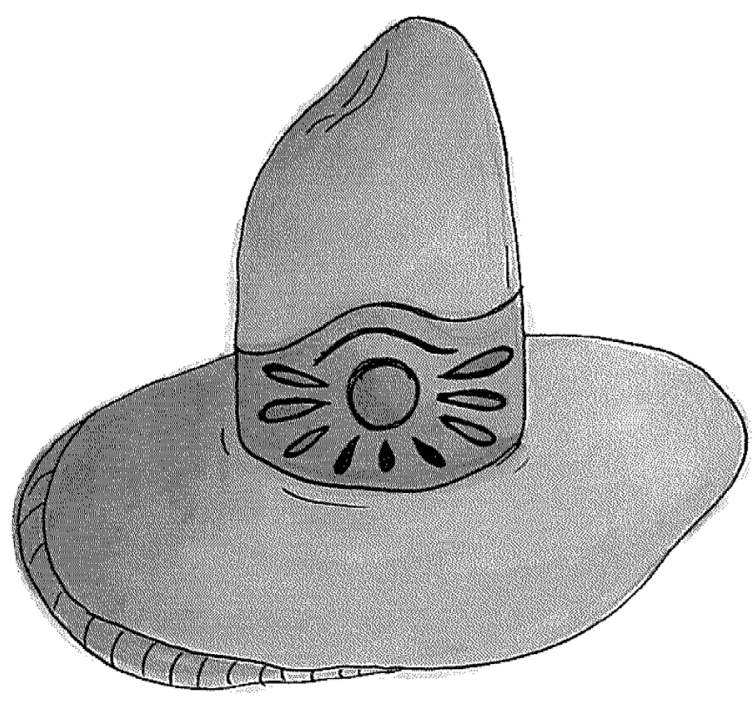

bajo. Una de las observaciones fue la falta de formación y conocimiento del tema por parte del asesor de titulación y carencia de tiempo para la atención. Los alumnos consideran que faltó abordar la formación de investigación y seguimiento constante del trabajo (no sólo durante los TVC). Al respecto, se sugirió que los asesores deben cuidar el proceso de avance de cada asesorado para concluir en tiempo y forma.

$\mathrm{Al}$ indagar acerca del aporte de la formación profesional para el desempeño de las funciones del puesto que ocupan, las respuestas de los egresados permitieron observar que van desde los conocimientos teóricos y prácticos, hasta aptitudes y actitudes que les permitieron una formación integral, favoreciendo así su desempeño en un amplio espectro de actividades en el ámbito laboral o el autoempleo.

En relación con los conocimientos teóricos adquiridos que aportaron a su desenvolvimiento laboral, mencionaron: vinculación comunitaria, experiencia en el área de investigación, elaboración de proyectos y búsqueda de información. Los conocimientos prácticos que reconocen como aportaciones importantes fueron: control de grupos, prácticas de campo, capacitación, conocimiento de aves y fauna, kayak y nudos (manejo de cuerdas).

El modelo intercultural de la UIMQROo pretende "incorporar actores comunitarios en el proceso de construcción y sistematización del conocimiento y en el tratamiento profesional de los problemas así como en la búsqueda de soluciones" (UIMQRoo, 2010). A su vez, introduce a los egresados al entendimiento de la sociedad, brindando también competencias en comunicación y expresión, desarrollando valores como la ética, responsabilidad y compromiso, liderazgo, trabajo en equipo, resolución de consensos, calidad y servicio al cliente. Por último, el PE de la licenciatura en Turismo Alternativo fomenta la identificación de necesidades y del potencial que se tiene para el desarrollo de actividades turísticas (UIMQRoo, 2016).

$\mathrm{Al}$ indagar acerca de la pertinencia de las asignaturas impartidas con su aplicación a la vida profesional, se preguntó a los egresados cuáles consideran útiles para su formación como licenciados en Turismo Alternativo. Mencionaron el Taller de Elaboración y Evaluación de Proyectos, Flora y Fauna de Interés Turístico, Manejo y Control de Grupos y Arqueología. También se contrastó con las asignaturas que generaron dificultades para vincular su contenido con la realidad de su trabajo y éstas fueron: Estadística, Elaboración de Proyectos e Inglés.

Asimismo, se mencionaron 34 asignaturas que "necesitan" durante su desempeño profesional y que no se encuentran en el PE; las más referidas están relacionadas con la economía, la administración, la pedagogía y los idiomas; y que las asignaturas de investigación son necesarias para continuar con los estudios de maestría.

\section{Proceso de enseñanza-aprendizaje}

Se preguntó a los egresados si las actividades realizadas durante las clases fueron útiles para las funciones que realizan en su empleo: $74 \%(\mathrm{n}=17, \mathrm{~N}=23)$ contestó que sí, $9 \%$ contestó negativamente y $17 \%$ no contestó. Se argumentó que las dinámicas aplicadas por los profesores de inglés y las estrategias de manejo y control de grupos las aplican en sus trabajos: dando clases, así como técnicas de rapel, tirolesa y nudos. También son útiles en su desenvolvimiento las técnicas de rescate y resolución de conflictos, conocimientos básicos de marketing y elaboración de proyectos. Las prácticas de historia de Yucatán y arqueología son esenciales en su desempeño. 
En relación con las prácticas de campo, 91\% $(\mathrm{n}=21, \mathrm{~N}=23)$ opinaron que son útiles como experiencia para el trabajo y sólo $9 \%$ no contestó $(n=2)$.

$\mathrm{Al}$ indagar sobre qué actividades de aprendizaje consideran más significativas para resolver las tareas en su empleo actual, los egresados mencionaron las señaladas en la Gráfica 1. Además, indicaron: dinámicas, investigación, guías, comunicación, viajes de prácticas, planeación y logística.

GráfICA 1

Actividades consideradas importantes y útiles por su aplicación en el desempeño profesional

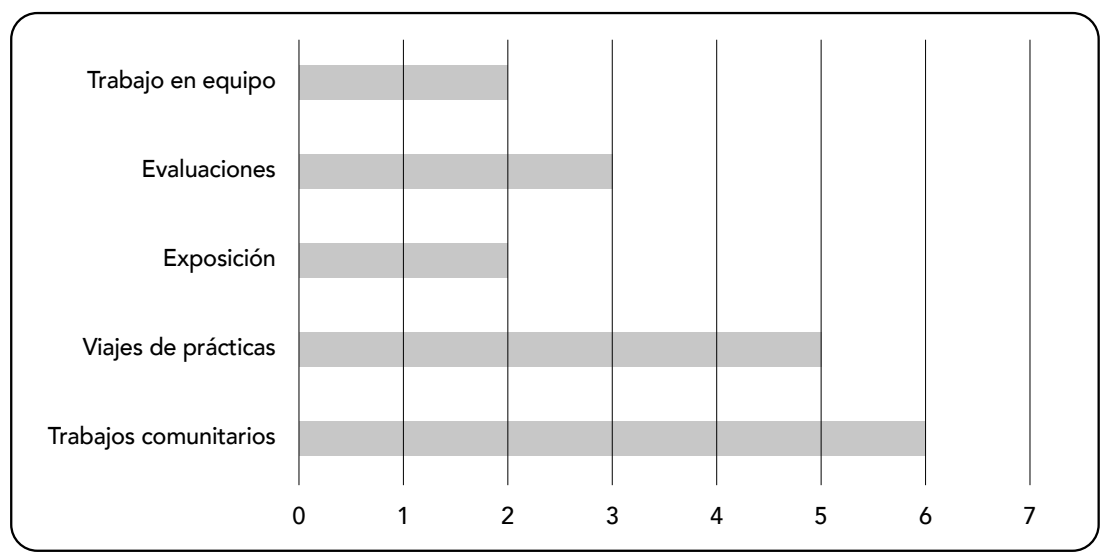

\section{Procedimiento de evaluación}

A partir de su experiencia, pedimos a los egresados que proporcionaran ejemplos que ilustraran la manera en que las evaluaciones en las diferentes asignaturas aportaron para simular situaciones semejantes a las tareas que realizan en su actual o anterior empleo. Los resultados fueron que las presentaciones en aula, los proyectos, las propuestas, las salidas de campo y la convivencia con la comunidad les ayudaron vinculando las asignaturas a ciertos casos.

Los egresados consideran que las prácticas de campo ayudaron a identificar las características o condiciones del ambiente natural; las prácticas simularon la atención al cliente; la comunicación verbal a perder miedo para hablar frente al público y grupos, ya que les proporciona seguridad para enseñar (exposición frente a grupo, exámenes, control de grupo).

Los rallies, por su parte, crearon el ambiente de organización y coordinación, simulando las etapas que comprende un proyecto, desde la planeación hasta la implementación, pues estas actividades son muy completas, y la organización, la logística y aplicación son responsabilidad de los estudiantes.
Se les preguntó a partir de su experiencia como estudiantes cuál fue la retroalimentación que los profesores dieron a su desempeño en las diferentes actividades de evaluación, a lo que calificaron como claras, concisas y pertinentes. Desde el punto de vista didáctico, recomendaron que cada profesor tenga un expediente de avance y mejora del estudiante, que permita conocer los estilos de aprendizaje del alumno.

\section{Perfil del docente (trabajos en equipo}

para discutir y proponer situaciones ideales de los temas abordados)

Ante la pregunta sobre el perfil del docente, 74\% contestó que los profesores sí cuentan con este perfil, mientras 13\% respondió negativamente e igual cantidad no contestó.

\section{Taller de vinculación con la comunidad}

Los egresados aportaron sugerencias para mejorar los TVC: una de éstas fue formar grupos multidisciplinarios que atiendan una problemática real de la comunidad y realicen en conjunto el análisis FODA (Fortaleza, Oportunidad, Debilidad y Amenaza); 
otra sugerencia es que los talleres de preparación no sean sólo en dos semanas antes del verano. De igual manera, se sugirieron más asesorías y prácticas con los estudiantes, agregar prácticas o estadías profesionales.

\section{Servicio social}

Otro de los puntos evaluados fue la aportación del servicio social como experiencia significativa relacionada con las actividades profesionales que realiza el egresado en la actualidad: $74 \%$ contestó la interrogante $(n=17, N=23)$, mientras $26 \%$ no respondió a las preguntas del cuestionario. La valoración se realizó en tres escalas: mucho (65\%), poco (18\%) y nada $(17 \%)$. Al aportar sus sugerencias, lo más sobresaliente fue que el servicio social sea en empresas turísticas y la comunicación con extranjeros (idiomas). Entre los que contestaron en negativa, sobresale que no era muy evidente el que hayan realizado un servicio social al estar inmersos en los veranos, y era notoria la falta de preparación al trabajar con grupos comunitarios.

\section{Intercambio académico}

Los egresados que participaron en los programas de intercambio expresaron que esta experiencia los ayudó para seguir estudiando, generó un vínculo académico con el asesor y los profesores que los recibieron. Las experiencias que adquirieron fueron principalmente el ser líder y ayudar a la solución de problemas, desarrollo de nuevas competencias, mejora en su visión intercultural, diseño de proyectos, valoración del idioma del mundo y experiencia personal. En relación al trámite, sólo un alumno de los ocho participantes mencionó que fue inadecuado; los siete restantes lo calificaron de excelente a muy bueno. A su vez, dos estudiantes manifestaron que posterior a su intercambio no existió un seguimiento durante su estadía en la institución receptora.

Los egresados que no participaron en un intercambio académico manifestaron que los motivos fueron: falta de tiempo e interés; se enteraban a último momento, no contaban con el promedio; que a pesar de participar no fueron elegidos, ya que eran pocas convocatorias; para no atrasar el proceso de titulación; falta de asesoramiento y motivación; o por adeudar asignaturas.

Entre las acciones sugeridas para mejorar el programa están: considerar a los egresados; que los profesores generen el interés en sus alumnos; hacer conferencias con las personas que han salido de intercambio para compartir experiencias y promoción; dejar que los alumnos concursen aunque no cumplan con el promedio; más convenios con otras universidades de Latinoamérica enfocadas al turismo alternativo; gestión de apoyos para que los estudiantes realicen estos intercambios; flexibilidad en los veranos; creación de un programa de preparación del idioma para intercambios internacionales; inclusión de los estudiantes en los programas de intercambios docentes; hacer reuniones informativas e identificación de instancias con asignaturas o carga curricular similares para validar durante la estancia.

\section{Consideraciones finales}

El crecimiento del turismo alternativo es tal que el gobierno y la iniciativa privada hacen esfuerzos para dotar de infraestructura y capacitación con el objetivo de atender adecuadamente este sector. En el estado de Quintana Roo, por su cualidad turística, varias universidades brindan programas educativos en materia turística, aunque sólo la UIMQROO ofrece en forma integral el nivel de profesional asociado y la licenciatura en Turismo Alternativo, además del enfoque educativo intercultural.

El reto a superar es ofrecer una formación de calidad para cubrir varios aspectos. En el mundo, incluido México, se han reportado casos exitosos de turismo alternativo comunitario, que iniciaron con el mercado interno y crecieron hasta lograr una buena reputación internacional. Lo han hecho con base en la planeación participativa comunitaria, con organización, buenas técnicas de solución de conflictos, visión y misión.

Al considerar todo lo anterior, resulta evidente la necesidad de la inmersión de los egresados con los perfiles a nivel profesional para coadyuvar a la solución de estos problemas regionales. La finalidad del

48 - La licenciatura en Turismo Alternativo de la Universidad Intercultural Maya de Quintana Roo (UIMQROO)... Cecilia del Socorro Medina Martín, Margarita de Abril Navarro Favela, Andrea Danilu Uc Angulo. DIDAC 76 (2020): 40-50 
PE de Turismo Alternativo no se reduce sólo al aspecto de los ingresos económicos, sino que se vuelve necesario un análisis del impacto en los ámbitos sociales, culturales, políticos y lingüísticos vinculados con las comunidades mayas. Es indispensable realizar una detección de sus problemas y demandas sociales, así como la inserción laboral de los egresados. Esto permitirá evaluar la pertinencia y la intervención profesional de la licenciatura en el desarrollo socioeconómico de la región.

$\mathrm{Al}$ concluir el análisis del cuestionario y de los grupos focales con los egresados, fue posible saber que la mayoría de ellos están laborando en la prestación de algún servicio turístico en la región, mientras que otros se desempeñan en la docencia y que el modelo intercultural de la UIMQROO contribuyó en las competencias, habilidades y capacidades para cumplir con sus funciones en el área laboral.

Los TVC realizados en los veranos favorecen la elaboración de proyectos sociales, el conocimiento de trabajar y solventar las necesidades que guardan las poblaciones locales mediante la organización de ellas, y lograr acuerdos a través del consenso. Los TVC también contribuyen en la adquisición de capacidades de redacción de informes y proyectos.

Las lenguas maya, inglesa y la interculturalidad les ayudaron a realizar aportaciones en su empleo actual desde distintas áreas; prueba de ello es la experiencia y el aprecio por parte de sus empleadores. Las competencias necesarias para ejercer la profesión son los idiomas: los egresados mencionaron que saber o no varios idiomas es una fortaleza o limitante, según sea el caso, al momento de conseguir un empleo, ascensos, mejores trabajos o continuar a un posgrado.
En la evaluación curricular, se destacó que en los primeros semestres se inclina hacia las asignaturas biológicas y se recomendó la impartición de asignaturas de la segmentación del turismo (de aventura, de naturaleza, cultural y agroturismo), para que los alumnos, desde el inicio de la carrera, puedan elegir sus trabajos de tesis.

Otra de las recomendaciones está focalizada en los TVC, para que en ellos los estudiantes realicen diagnósticos centrados en turismo. Los egresados externaron que una de sus limitaciones en la contratación de empleos son las certificaciones en la NOM, ya que los empleadores las solicitan como requisito previo.

En cuanto a la experiencia adquirida durante la carrera, los egresados coinciden en que las prácticas tienen concordancia con las situaciones reales de trabajo; sin embargo, las evaluaciones deberían ser equitativas en lo teórico y práctico. Reconocieron la desventaja que enfrentan al no realizar prácticas profesionales en empresas o centros turísticos, ya que no desarrollan las competencias de esta experiencia laboral.

Asimismo, reconocen que el modelo educativo los preparó respecto a las aptitudes y actitudes, al tener como base la vinculación comunitaria con el enfoque de interculturalidad. Las aptitudes y actitudes son reconocidas por los empleadores y facilitan la interacción con sus compañeros y clientes, al respetar la diversidad, el intercambio y enriquecimiento mutuo de personas de culturas distintas (UIMQROO, 2016). Los egresados externaron que el modelo les proporcionó competencias que contribuyeron a su formación hoy en día como profesionistas.

REFERENCIAS

Alvarado, L. \& García M. (2008). Características más relevantes del paradigma socio-crítico: su aplicación en investigaciones de educación ambiental y de enseńanza de la ciencias realizadas en el doctorado de Educación en el Instituto Pedagógico de Caracas. Sapiens. Revista Universitaria de Investigación, 9(2), 187-202.
Casillas, M. de L. \& Santini, L. (2009). Universidad intercultural. Modelo educativo. México: CGEIB-SEP.

Fernandez, S. (1995). Consideraciones sobre la teoría socio-crítica de la enseñanza. Enseñanza, 13, 241-259.

García Retana, A. (2011). Modelo educativo basado en competencias. Importancia y necesidad. Revista Electrónica Actua- 
lidades Investigativas en Educación, 11(3), 1-24. Recuperado de https://www.redalyc.org/pdf/447/44722178014.pdf

Maldonado, J. (2018). Metodología de la investigación social. Paradigmas: cuantitativo, sociocrítico, cualitativo, complementario. Bogotá: Ediciones de la U.

Melero, N. (2011). El paradigma crítico y los aportes de la investigación acción participativa en la transformación de la realidad social: un análisis desde las ciencias sociales. Cuestiones Pedagógicas, 21, 339-355.

Navarro, M. (2015). Acción y reflexión para mejorar la eficacia de la tutoría en una universidad intercultural (Tesis doctoral). Universidad Anáhuac Mayab, Mérida, Yucatán.

Philipp, R. (1998). La teoría del actuar comunicativo de Jürgen Habermas: un marco para el análisis de las condiciones socializadoras en las sociedades modernas. Papers, 56, 103-123.

Saldívar, A. (Coord.). (2006). Técnicas y dinámicas para la Educación Intercultural, México: Ecosur, Instituto Austriaco de Cooperación Norte Sur, Casa de la Ciencia.

Sánchez, A. (2016). La educación intercultural como principal modelo educativo para la integración social de los inmigrantes. Cadernos de Dereito Actual, 4, 139-151.
Schmelkes, S. (2008). Las universidades interculturales en México: ¿una contribución a la equidad en educación superior? Recuperado de https://www.academia.edu/3048258/ LAS_UNIVERSIDADES_INTERCULTURALES_ EN_M\%C3\%89XICO_Una_contribuci\%C3\%B3n_a_ la_equidad_en_educaci\%C3\%B3n_superior

Schmelkes, S. (2013). Educación para un México intercultural. Sinéctica, 40, 1-12. Recuperado de https://www.redalyc. org/pdf/998/99827467007.pdf

Universidad Intercultural Maya de Quintana Roo (UIMQRoo) (2010). Modelo Educativo Intercultural. Recuperado de http://www.uimqroo.edu.mx/Documentos/NuestraUniversidad/Modelo-educativo-texto.pdf

Universidad Intercultural Maya de Quintana Roo (UIMQROo) (2015). Guía para el taller de vinculación con la comunidad I, II y II. Documento inédito.

Universidad Intercultural Maya de Quintana Roo (UIMQRoo) (2016). Programa de Desarrollo Institucional, Universidad Intercultural Maya de Quintana Roo 2016-2020.

\section{Cecilia del Socorro Medina Martín. Licenciada en Ciencias Antropológicas con especialidad en Arqueología por la Facultad de Ciencias Antropológicas, con especialización y maestría en Antropología Esquelética por la Universidad Autónoma de Yucatán (UADY). Actualmente se desempeńa como profesora investigadora de tiempo completo en la carrera de Turismo Alternativo de la Universidad Intercultural Maya de Quintana Roo (UIMQROo). Cuenta con una amplia participación como ponente en congresos nacionales e internacionales y ha escrito varios artículos y capítulos en libros de patrimonio, arqueología y temas afines.}

SemblanZas

Margarita de Abril Navarro Favela. Doctora en Ciencias de la Educación. Desde 2009 es profesora investigadora en la UIMQROO; realiza las funciones sustantivas de investigación, vinculación y docencia por lo que, desde 2012, conserva el reconocimiento como profesora con Perfil Deseable prodep que otorga la Secretaría de Educación Pública (sEP) y cuenta con la distinción de Candidata en el Sistema Nacional de Investigadores (sNI) del Conacyt. Es responsable del proceso de investigación en la UIMQROo e integrante del Grupo Técnico de Fomento a la Investigación, Ciencia, Tecnología e Innovación en la Comisión Estatal para la Planeación de la Educación Superior (coepes) de Quintana Roo. Como parte de su compromiso social también dirige proyectos de desarrollo comunitario, es mediadora de lectura y trabaja con los grupos de turismo rural. En marzo de 2019 recibió la distinción de Mujer Quintanarroense Destacada en el ámbito turístico.

Andrea Danilu Uc Angulo. Maestra en Planificación de Empresas y Desarrollo Regional y licenciada en Turismo Alternativo. Tiene cuatro ańos de experiencia docente y es profesora en la Universidad Tecnológica Metropolitana (UTM) de Mérida, Yucatán. Ha colaborado en proyectos enfocados al desarrollo turístico y cultural de la península de Yucatán, como el

"Diagnóstico para la identificación de productos de las Comunidades de Alto Valor

Comercial y Nichos de Mercado Alternativo" y "Diagnóstico de accesibilidad de los recursos de la ruta de la guerra de castas". Por otra parte, ha participado como ponente en diversos congresos nacionales e internacionales y también ha asesorado proyectos universitarios.

50 - La licenciatura en Turismo Alternativo de la Universidad Intercultural Maya de Quintana Roo (UIMQROO).. 